\title{
Multi-parametric Dynamic Analysis of a Rolling Bearings System
}

\author{
Almatbek Kydyrbekuly ${ }^{1}$ - Gulama-Garip Alisher Ibrayev ${ }^{1}$ - Tangat Ospan ${ }^{1}$ - Anatolij Nikonov2,* \\ 1 Al-Farabi Kazakh National University, Kazakhstan \\ 2 Faculty of Industrial Engineering Novo mesto, Slovenia
}

A method for calculating amplitudes and constructing frequency characteristics of forced and self-excited vibrations of a rotor-fluid-foundation system on rolling bearings with a non-linear characteristic based on the method of complex amplitudes and harmonic balance has been developed. Non-linear equations of motion of the rotor-fluid-foundation system are derived, and analytical methods of their solution are presented. Frequencies of fundamental and ultra-harmonic resonances are determined. The intervals between self-oscillation frequencies are estimated. The dependence of amplitudes on the amount of fluid in the rotor cavity, the mass of the foundation, linear imbalance, the value of the stiffness coefficient, and the damping coefficient is shown.

Keywords: forced vibrations, resonance, fluid, rolling bearing, self-oscillations, ultra-harmonic

\section{Highlights}

- A dynamic model of the rotor-fluid-foundation system on rolling bearings with a non-linear characteristic has been constructed.

- The specific features of the system with many degrees of freedom are defined.

- The prerequisites under which the fluid stabilizes the behaviour of a non-linear system are determined.

- The optimal parameters of the system, providing its stable operating mode, are determined.

\section{O INTRODUCTION}

One of the main tasks of dynamics of rotors and design of rotary machines is the choice of parameters that provide their stable operation. The damping of forced and purely non-linear self-excited rotor vibrations by selecting parameters of the system, taking into account vibrations of the foundation, elasticity of supports, imbalance and other parameters, is of great interest from both technical and economic points of view.

Currently, many works on the study of nonlinear oscillations of rotor systems exist [1]. However, their dynamics is insufficiently studied because of difficulties arising in taking into account the mutual influence and combined actions of factors such as the presence of fluid in the rotor cavity, external nonconservative forces [2], different types of linear and angular imbalances and nonlinearities [3] and [4].

In the design of rotary machines, one of the most important mechanical components described by nonlinear models is elastic support, which ensures the operability and reliability of the system [5] to [7]. In this paper, rolling bearings act as elastic supports [8]. The neglect of non-linear properties of bearings prevents correct qualitative and quantitative results for rotor systems from being obtained [9] to [11]. This phenomenon can be explained by the fact that when analysing linear rotor systems with rolling bearings, an approximate estimate of stiffness and damping properties of bearings is most often used, whereas in reality the bearing stiffness heavily depends on the load, i.e., on the operating mode of the rotor system, on the geometry and size of the bearing clearances, on the size of the fit of the inner and outer rings in the bearing, and other factors [12] and [13].

To obtain a detailed description of the process, it is necessary to consider the influence of such factors as imbalance, asymmetry of the rotor on the shaft, external friction, changes in inertial parameters and positional forces of various kinds [14] to [16]. Such complications of the model in the analysis of dynamics make it possible to study the influence of the gap size, rotation frequency on frequency spectra, and amplitude-frequency characteristics for any rotor system on rolling bearings.

Mathematical models of bearings that take into account nonlinearity factors are distinguished by complexity and primarily by the loads that they consider. In our case, to describe the bearing model, the Hertz contact theory is used, which connects the radial loads acting on the bearing and the deformation at the contact points between the rolling element and the bearing rings [17]. In the bearing model, it is assumed that there are no types of sliding of bodies and rolling surfaces. Damping is considered in terms of equivalent viscous and linear friction.

\section{STATEMENT OF PROBLEM AND EQUATIONS OF MOTION}

A symmetrical vertical rotor of mass $m$ with a cylindrical cavity of radius $R$, partially filled with an ideal fluid, is rotating on rolling bearings with a constant angular velocity $\Omega_{0}$. The angular velocity is considered sufficiently large so that the fluid in the 
rotor cavity takes the form of a cylindrical ring. Due to the different positions of the centre of mass and the geometric centre, the rotor has a static imbalance (or linear eccentricity) $e$. The foundation of mass $M$ rigidly connected to the outer ring is mounted on elastic supports with an equivalent linear stiffness coefficient $c_{2}$ (see Fig. 1); below, all the dimensional parameters shown in this figure are appropriately dimentionalized, see Section 3. The radial compliance of bearings occurs due to deformation of the rolling elements and raceways at the contact points. In this case, the non-linear restoring force in bearings can be generally described using the Hertz contact formula [14] and [18]:

$$
F_{C}=C_{b} \delta_{r}^{\frac{3}{2}}
$$

However, for the sake of simplicity, in what follows, the restoring force in rolling bearings is approximated by a power series according [16]:

$$
F_{C}=c_{0} \delta_{r}+c_{1} \delta_{r}^{3},
$$

where $F_{C}$ is a component of the restoring force in the radial direction, $\delta_{r}$ is the deformation in the radial direction, $C_{b}$ is the stiffness coefficient, $c_{0}$ and $c_{1}$ are stiffness coefficients for the linear and cubic terms, respectively. This expansion at $\delta_{r}<1000 \mu \mathrm{m}$ agrees with the experimental results in [18].

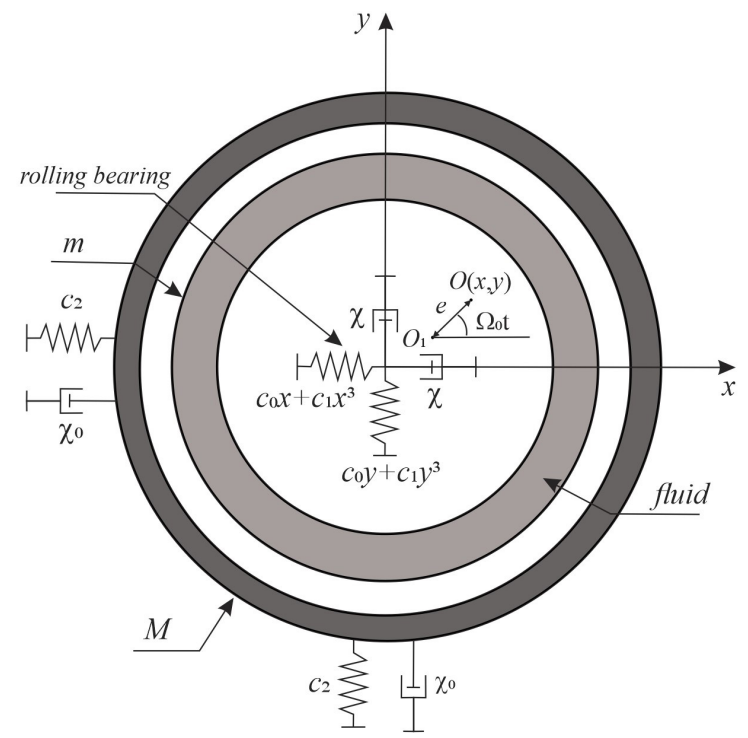

Fig. 1. Scheme of a rotor on rolling bearings, with a cavity partially filled with an ideal fluid and mounted on a foundation

The motion of the system is considered concerning the fixed coordinate system Oxyz. The coordinates of the centre of mass of the rotor are denoted as $A(x, y)$, and the coordinates of the centre of mass of the foundation are denoted as $A_{2}\left(x_{2}, y_{2}\right), \chi$ and $\chi_{0}$ are the external friction coefficients, $\mathrm{O} \xi \eta$ the coordinate system associated with the rotor, $\eta$ is its polar axis, and axis $\xi$ is arbitrarily drawn through the eccentricity vector of the rotor mass.

Let us introduce complex variables in the form:

$$
x+i y=z, \quad x_{2}+i y_{2}=z_{2},
$$

for analysing plane-parallel motion of the rotor and foundation. Then, assuming that damping force acting on rotor depends on velocities of the rotor itself, and similarly, damping force acting on foundation depends on the velocities of the foundation itself, we present the equations of motion of the system in question in the form, here and below see [8] and references therein,

$$
\begin{aligned}
& \ddot{z}+n_{0}^{2}\left(z-z_{2}\right)+n_{1}\left(z-z_{2}\right)^{3}+2 k \dot{z}= \\
& =e \Omega_{0}^{2} \exp \left(i \Omega_{0} t\right)+\frac{F_{r}}{m}, \\
& \ddot{z}_{2}+n_{2}^{2} z_{2}-n_{01}^{2}\left(z-z_{2}\right)-n_{10}\left(z-z_{2}\right)^{3}+2 k_{0} \dot{z}_{2}=0,
\end{aligned}
$$

where

$$
n_{0}^{2}=\frac{2 c_{0}}{m}, n_{1}=\frac{2 c_{1}}{m}, 2 k=\frac{\chi}{m}, n_{2}^{2}=\frac{2 c_{2}}{M},
$$

$n_{01}^{2}=\frac{2 c_{0}}{M}=\mu n_{0}^{2}, n_{10}=\frac{2 c_{1}}{M}=\mu n_{1}, 2 k_{0}=\frac{\chi_{0}}{M}, \mu=\frac{m}{M}$,

and $F_{r}$ is a complex expression for the reaction force of a fluid, which is defined as:

$$
F_{r}=\left.R h \int_{0}^{2 \pi} P\right|_{r=R} e^{i\left(\Omega_{0} t+\varphi\right)} d \varphi,
$$

where $h$ is the rotor cavity height, $\left.P\right|_{r=R}$ is the fluid pressure on the rotor wall.

The equations of motion for fluid are written as:

$$
\begin{aligned}
\frac{\partial u}{\partial t}-2 \Omega_{0} v & =-\frac{1}{\rho} \frac{\partial P}{\partial r}-\ddot{x} \cos \left(\Omega_{0} t+\varphi\right) \\
& -\ddot{y} \sin \left(\Omega_{0} t+\varphi\right), \\
\frac{\partial v}{\partial t}+2 \Omega_{0} u & =-\frac{1}{\rho r} \frac{\partial P}{\partial \varphi}+\ddot{x} \sin \left(\Omega_{0} t+\varphi\right) \\
& -\ddot{y} \sin \left(\Omega_{0} t+\varphi\right),
\end{aligned}
$$

where $u$ and $v$ are the radial and tangential components of the velocity of the fluid particle, $P$ and $\rho$ are pressure and density of the fluid.

The continuity equation at $\rho=$ const is given by:

$$
\frac{\partial(u r)}{\partial r}+\frac{\partial v}{\partial \varphi}=0
$$


The boundary conditions for the Eqs. (6) and (7) can be written as:

$$
\left.u\right|_{r=R}=0
$$

and

$$
\left.\frac{\partial P}{\partial t}\right|_{r=r_{0}}=-\left.\rho \Omega_{0}{ }^{2} r_{0} u\right|_{r=r_{0}},
$$

where $r_{0}$ is the radius of the free fluid surface. These relations express the impenetrability condition at the rotor wall and the conditions on the fluid pressure along the free surface, respectively.

Eqs. (3) to (7) with boundary conditions Eqs. (8) and (9) in combination with the continuity equation form a closed system of equations.

\section{NON-LINEAR FORCED VIBRATION AND SELF- OSCILLATIONS OF THE ROTOR-FLUID-FOUNDATION SYSTEM}

Assuming that the rotor and the foundation perform harmonic oscillations, we seek the solution of the system of equations Eq. (4) in the form [20] and [21],

$$
\begin{gathered}
z=Z_{r} \exp \left(i\left(\Omega_{0} t-\phi\right)\right)+A_{r} \exp (i \omega t), \\
z_{2}=Z_{f} \exp \left(i\left(\Omega_{0} t-\phi\right)\right)+B_{f} \exp (i \omega t),
\end{gathered}
$$

where $\omega$ is natural frequency, $Z_{r}$ and $Z_{f}$ are forced vibration amplitudes of rotor and foundation, while $A_{r}$ and $B_{f}$ are natural vibration amplitudes of rotor and foundation, respectively.

The presence of an angle $\phi$ i.e., a phase lag, is caused by the presence of resistance forces in the system.

As an expression describing the action of the hydrodynamic force on the system, which arises due to the presence of fluid in the cavity, we start from formulae in [8] and [19], taking into account Eqs. (10) and (11):

$$
\begin{aligned}
F_{r}= & F_{x}+i F_{y}=Z_{r} m_{L} \Omega_{0}{ }^{2} \exp \left(i\left(\Omega_{0} t-\phi\right)\right)+ \\
& +A_{r} m_{L} \omega^{2} \frac{\left(\sigma^{2}-2 \Omega_{0} \sigma-\Omega_{0}{ }^{2}\right)}{\left(\gamma \sigma^{2}-2 \Omega_{0} \sigma-\Omega_{0}{ }^{2}\right)} \exp (i \omega t),
\end{aligned}
$$

where

$$
q=\frac{R}{r_{0}}, \quad \sigma=\omega-\Omega_{0},
$$

$m_{L}=\pi \rho R^{2} h$ is the mass of fluid required to completely fill the rotor cavity, $\sigma$ vibration frequency of the free surface of the fluid.
Substituting Eqs. (10), (11) and (12) in Eq. (4), we obtain a system of algebraic equations for the unknowns $A_{r}, B_{f}, Z_{r}$ and $Z_{f}$. Its solution is given by:

$$
\begin{gathered}
Z_{r}=\frac{f_{0}}{f_{1}}, \quad Z_{f}=\frac{f}{f_{1}}, \\
B_{f}=\left(p_{1}+i p_{2}\right) A_{r}, \\
Z_{r}-Z_{f}=\frac{f_{0}-f}{f_{1}}=f_{2}, \\
A_{r}=\left(\frac{p_{1}}{p^{2}{ }_{1}+p_{2}^{2}}-\frac{i p_{2}}{p^{2}{ }_{1}+p_{2}{ }^{2}}\right) B_{f},
\end{gathered}
$$

where

$$
\begin{gathered}
f_{0}=r p_{0}-p r_{0}, \quad f=p q_{0}+q p_{0}, \quad f_{1}=r q_{0}+q r_{0}, \\
q=\mu \Omega_{0}^{2}\left(1+\mu_{L}\right), \quad p=\mu e \Omega_{0}^{2} \cos \phi, \quad r=n_{2}^{2}-\Omega_{0}^{2}, \\
q_{0}=2 \mu \Omega_{0} k, \quad p_{0}=\mu e \Omega_{0}^{2} \sin \phi, \quad r_{0}=2 k_{0} \Omega_{0}, \\
p_{1}=\frac{\mu \omega^{2}\left(n_{2}^{2}-\omega^{2}\right)\left(1+\mu_{L} \frac{D_{3}}{D_{4}}\right)-4 k k_{0} \omega^{2}}{m_{1}}, \\
p_{2}=-\frac{2 \mu\left(\left(1+\mu_{L} \frac{D_{3}}{D_{4}}\right) \omega^{2} k_{0}+\left(n_{2}^{2}-\omega^{2}\right) k\right) \omega}{m_{1}}, \\
m_{0}=n_{2}^{2}-\Omega_{0}^{2}+2 k_{0} \Omega_{0} i, \quad m_{1}=\left(n_{2}^{2}-\omega^{2}\right)^{2}+4 k_{0}^{2} \omega^{2}, \\
m_{2}=n_{2}^{2}-\omega^{2}+2 k_{0} \omega i, \quad D_{3}=\sigma^{2}-2 \Omega_{0} \sigma-\Omega_{0}^{2}, \\
D_{4}=\gamma \sigma^{2}-2 \Omega_{0} \sigma-\Omega_{0}^{2} .
\end{gathered}
$$

$\mu_{L}=m_{L} / m$ is the ratio of the mass of fluid required to completely fill the rotor cavity to the mass of the rotor.

Thus, to determine the unknown amplitudes, we may derive two non-linear equations from the relations above:

$$
\begin{aligned}
& m_{0} Z_{f}-\left(Z_{r}-Z_{f}\right) \\
& \cdot\left(n_{01}+n_{10}\left(\frac{3}{4}\left(Z_{r}-Z_{f}\right)^{2}+\frac{3}{2}\left(A_{r}-B_{f}\right)^{2}\right)\right)=0, \\
& m_{2} B+\left(A_{r}-B_{f}\right) \\
& \cdot\left(-n_{01}-n_{10}\left(\frac{3}{2}\left(Z_{r}-Z_{f}\right)^{2}+\frac{3}{4}\left(A_{r}-B_{f}\right)^{2}\right)\right)=0 .
\end{aligned}
$$

After substituting Eqs. (14) to (16) into Eq. (18), a quadratic equation for $A_{r}$ can be obtained. Using the values of the amplitudes $Z_{r}, Z_{f}, A_{r}$ and $B_{f}$ calculated from Eq. (19), we can determine the phase angle $\phi$.

The linearized form of Eqs. (18) and (19) becomes: 


$$
\begin{gathered}
\ddot{z}+n_{0}^{2}\left(z-z_{2}\right)+2 k \dot{z}=0, \\
\ddot{z}_{2}+n_{2}^{2} z_{2}-n_{01}^{2}\left(z-z_{2}\right)+2 k_{0} \dot{z}_{2}=0 .
\end{gathered}
$$

Since the resistance forces do not strongly affect the value of the natural frequency [22], in this case, it is sufficient to find the frequency equation for $k=k_{0}=$ 0 , i.e., for the equation in the form:

$$
\left[\begin{array}{ll}
1 & 0 \\
0 & 1
\end{array}\right]\left[\begin{array}{c}
\ddot{z} \\
\ddot{z}_{2}
\end{array}\right]+\left[\begin{array}{cc}
n_{0}^{2} & -n_{0}^{2} \\
-\mu n_{0}^{2} & n_{2}^{2}+\mu n_{0}^{2}
\end{array}\right]\left[\begin{array}{c}
z \\
z_{2}
\end{array}\right]=\left[\begin{array}{l}
0 \\
0
\end{array}\right] .
$$

To obtain a frequency equation, we seek the solution in the form:

$$
z=z_{s} \exp (i(\omega t-\varphi)), z_{2}=z_{2 s} \exp (i(\omega t-\varphi)) .
$$

Substituting Eq. (22) into Eq. (21), we obtain:

$$
\begin{aligned}
& {\left[\begin{array}{cccc}
n_{0}^{2}-\omega^{2} & 0 & -n_{0}^{2} & 0 \\
0 & n_{0}^{2}-\omega^{2} & 0 & -n_{0}^{2} \\
-\mu n_{0}^{2} & 0 & n_{2}^{2}+\mu n_{0}^{2}-\omega^{2} & 0 \\
0 & -\mu n_{0}^{2} & 0 & n_{2}^{2}+\mu n_{0}^{2}-\omega^{2}
\end{array}\right]} \\
& \cdot\left[\begin{array}{c}
A_{s} \\
B_{s} \\
C_{s} \\
D_{s}
\end{array}\right]=\left[\begin{array}{l}
0 \\
0 \\
0 \\
0
\end{array}\right],
\end{aligned}
$$

where

$$
z_{s}=\sqrt{A_{s}^{2}+B_{s}^{2}}, \quad z_{2 s}=\sqrt{C_{s}^{2}+D_{s}^{2}} .
$$

Then, to find the values of the natural frequencies $\omega$, it is sufficient to find the determinant of system Eq. (23), i.e.:

$$
\operatorname{det}\left\|\begin{array}{cccc}
n_{0}^{2}-\omega^{2} & 0 & -n_{0}^{2} & 0 \\
0 & n_{0}^{2}-\omega^{2} & 0 & -n_{0}^{2} \\
-\mu n_{0}^{2} & 0 & n_{2}^{2}+\mu n_{0}^{2}-\omega^{2} & 0 \\
0 & -\mu n_{0}^{2} & 0 & n_{2}^{2}+\mu n_{0}^{2}-\omega^{2}
\end{array}\right\|=0 .(24)
$$

Then, the frequency equation is written as:

$$
\omega^{8}+\lambda_{1} \omega^{6}+\lambda_{2} \omega^{4}+\lambda_{3} \omega^{2}+\lambda_{4}=0,
$$

where

$$
\begin{gathered}
\lambda_{1}=-\left(\Delta_{1}+2 \Delta_{4}\right), \quad \lambda_{2}=\Delta_{1}^{2}-\Delta_{2} \Delta_{3}+4 \Delta_{1} \Delta_{4}+\Delta_{4}^{2}, \\
\lambda_{3}=-2 \Delta_{1} \Delta_{4}\left(\Delta_{1}+\Delta_{4}\right), \quad \lambda_{4}=\Delta_{1}^{2} \Delta_{4}^{2}-\Delta_{2}^{2} \Delta_{3}^{2}, \\
\Delta_{1}=n_{0}^{2}, \quad \Delta_{2}=-\mu n_{0}^{2}, \quad \Delta_{3}=-n_{0}^{2}, \quad \Delta_{4}=n_{2}^{2}+\mu n_{0}^{2} .
\end{gathered}
$$

Thus, four natural frequencies are obtained, where the lowest frequency is taken as the first frequency. The accuracy of the approximate formulae for natural frequencies are discussed in [19], [21], and [22].

It should be noted that the search for a solution in the form of Eqs. (10) and (11) makes it possible to study both forced and self-excited vibrations in general; however, at the same time, this approach excludes the possibility of studying steady-state vibrations (which is typical for the case of an empty or a completely filled rotor), since the partial filling of the cavity with fluid causes the appearance of selfoscillations.

Thus, by slowly changing the angular velocity of the rotor, it is possible to construct the amplitudefrequency characteristics of the system while varying the parameters of the rotor, foundation, and fluid.

\section{RESULTS AND DISCUSSION}

To analyse the system and determine the optimal operating mode, the amplitude-frequency characteristics of the rotor and foundation were constructed. They were obtained from Eqs. (18) and (19) and numerical solution of the differential equations of motion of the system for different degrees of filling of the rotor cavity with fluid, different values of the damping coefficient $(\chi)$, nonlinear stiffness $\left(c_{1}\right)$, imbalance $(e)$ and different values of the foundation mass $(M)$. For qualitative analysis of the system, the following dimensionless parameters were introduced:

$$
s=\frac{\Omega_{0}}{\omega}, \quad E=\frac{e}{R}, \quad K=\frac{k}{\Omega_{0}}, \quad C=\frac{n_{1} e^{2}}{\Omega_{0}^{2}},
$$

where $s$ is a dimensionless frequency, $E$ is a dimensionless parameter of linear imbalance, $Z_{r}$ is a dimensionless amplitude of the rotor, $Z_{f}$ is a dimensionless amplitude of the foundation, $K$ is a dimensionless damping coefficient, $C$ is a dimensionless coefficient of stiffness at the cubic term, $R$ is a cavity radius (see Figs. 2 to 11 ).

When an insignificant amount of fluid is present in the cavity, e.g. $r_{0}=0.93 R$, (see Figs. 2 and 3), three self-oscillation zones and three resonance amplitudes are observed for both the rotor and the foundation. All figures show examples when the system parameters take the values: $\chi=4200 \mathrm{~kg} / \mathrm{s}$, $\chi_{0}=6.59 \mathrm{~kg} / \mathrm{s}, c_{0}=1.1 \cdot 10^{7} \mathrm{~kg} / \mathrm{s}^{2}, c_{1}=0.87 \cdot 10^{7} \mathrm{~kg} / \mathrm{m}^{2} \mathrm{~s}^{2}$, $c_{2}=3.26 \cdot 10^{5} \mathrm{~kg} / \mathrm{s}^{2}, M=25 \mathrm{~kg}, m=2.4 \mathrm{~kg}, e=0.001 \mathrm{~m}$. The amplitude-frequency characteristic, in this case, demonstrates a monotonic increase over the interval $0<s<0.065$ up to the first resonance peak. The first resonance occurs at $s=0.065$, where the amplitudes are $Z_{r}=0.083$ and $Z_{f}=0.0091$. Then, over the interval $0.065<s<0.65$, a self-oscillating mode occurs (see Figs. 2 and 3) with maximum amplitudes of 0.028 for the rotor and 0.001 for the foundation, respectively. At $s=0.65$, the second resonance occurs with the 

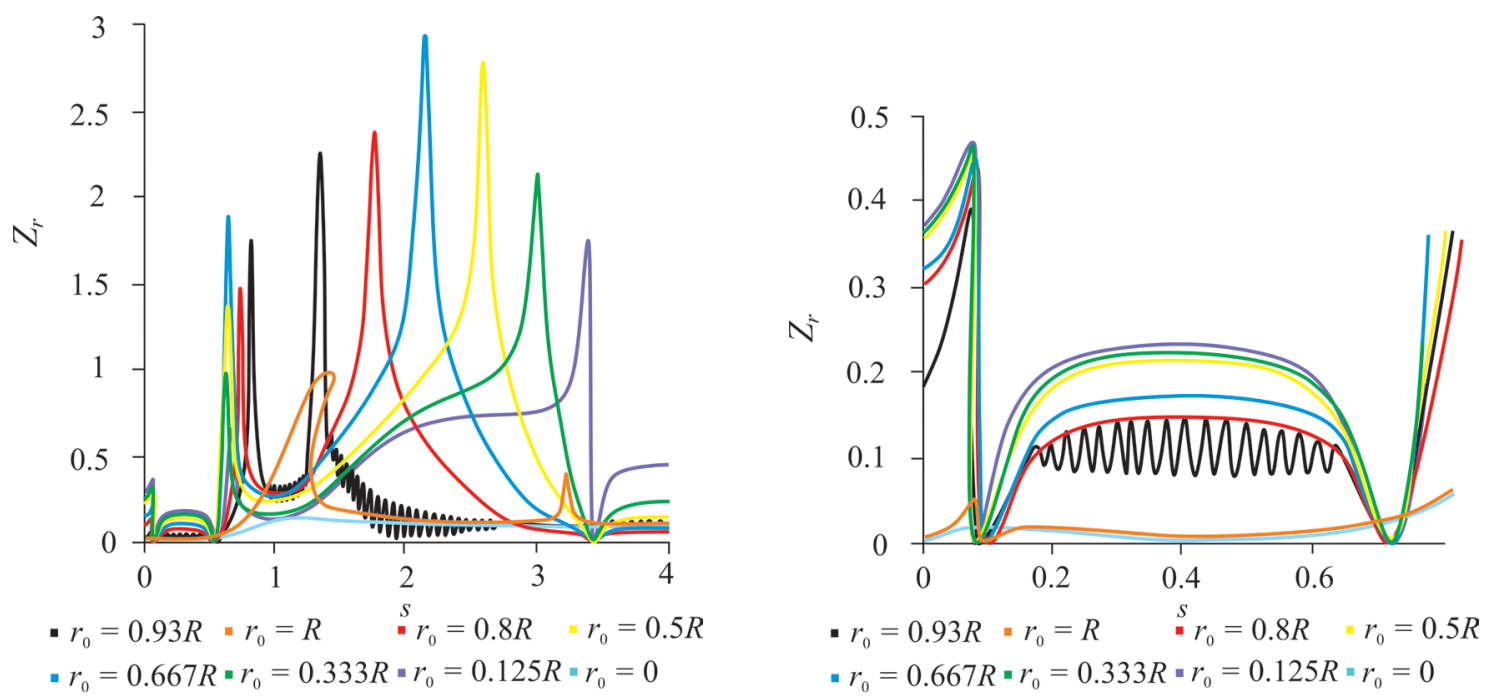

Fig. 2. Amplitude-frequency characteristics of the rotor at different degrees of the rotor cavity filling, $r_{0}$
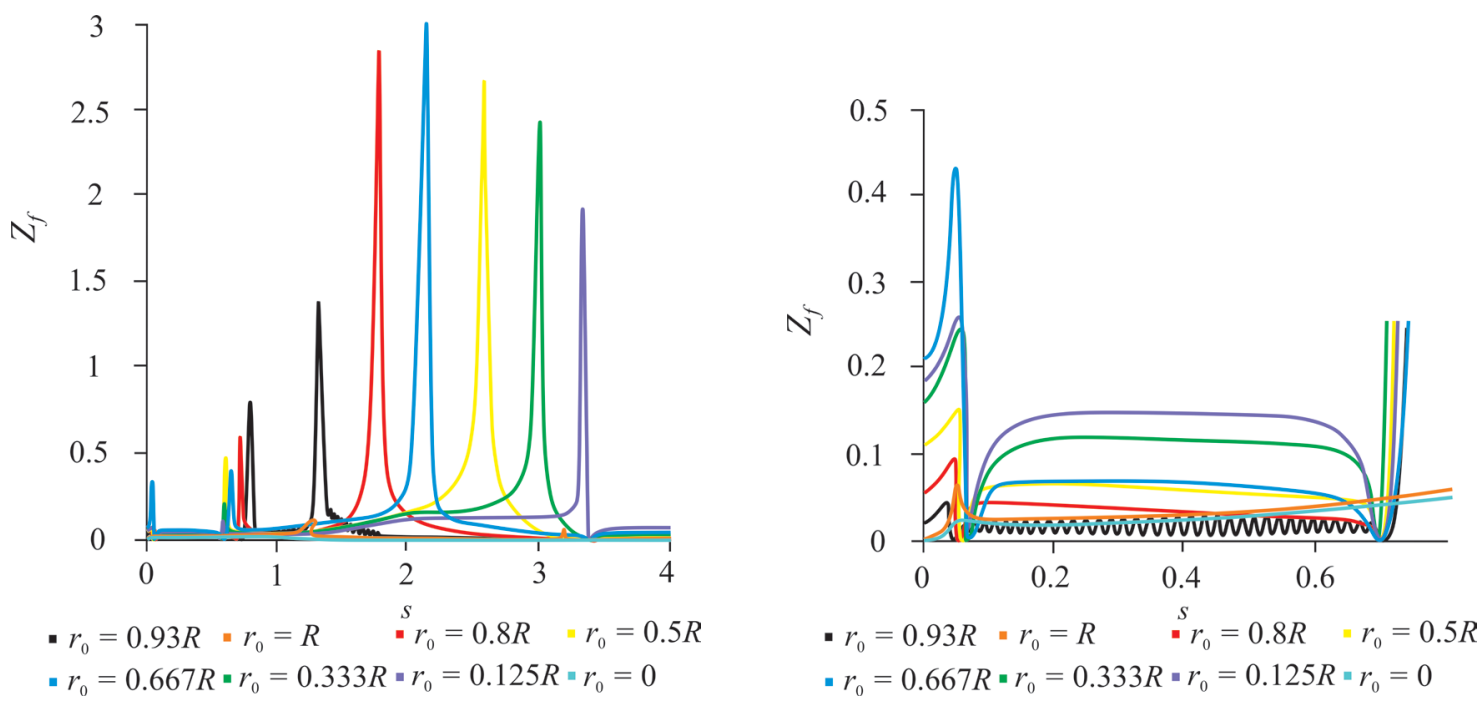

Fig. 3. Amplitude-frequency characteristics of the foundation at different degrees of the rotor cavity filling, $r_{0}$

amplitudes 1.72 and 0.79 for the rotor foundation, respectively. Further, over the interval $0.65<{ }_{S}<1$, the vibration amplitudes decrease monotonically to the values 0.07 and 0.013 . Next, over the interval $1<s<1.35$, the amplitude of vibrations grows rather intensively up to the third resonance, where $s=1.35$, and the amplitudes are $Z_{r}=2.23$ and $Z_{f}=1.38$. Further, in the interval $1.35<s<3.41$, the amplitudes monotonically decrease to the third self-oscillation zone, which occurs at $s=3.41$, where the amplitudes take constant values $Z_{r}=0.057, Z_{f}=0.0003$, which is caused by the effect of self-centring and nonconservative circulation-type forces arising due to the presence of fluid in the rotor cavity. Here, the self- oscillation zones are presented as a curve showing the oscillatory process. In all other cases, the curves corresponding to self-oscillations are smoothed.

With the increase of the fluid volume in the cavity at $r_{0}=0.8 R$, it can be seen that the first and second resonances, as well as the first self-oscillation zone, occur at the same frequencies as at $r_{0}=0.93 R$, but with larger amplitudes. For example, at the first resonance, i.e., at $s=0.065$, the amplitudes are $Z_{r}=0.15$ and $Z_{f}=0.015$, that is, a 4.74-times increase of the fluid volume leads to 1.82 - and 1.65-times increase in the amplitudes of the rotor and foundation, respectively. The maximum amplitudes of self-oscillations, in this case, also have higher values. For example, they reach 
0.088 for the rotor, and 0.0097 for the foundation, which is 3.14- and 9.7-times higher than in the previous case, respectively. At the second resonance $(s=0.65)$, the amplitudes are $Z_{r}=1.5$ and $Z_{f}=0.59$, which practically does not differ from the previous case. The third resonance peak in this case shifts to higher frequency range and occurs at $s=1.75$, where $Z_{r}=2.36, Z_{f}=2.83$. Then, for $s>3.41$, a self-oscillation zone is formed, where the amplitudes take on constant values $Z_{r}=0.139$ and $Z_{f}=0.0006$.

When the fluid volume in the rotor cavity is one third of the total volume, i.e., $r_{0}=0.667 R$ (see, e.g., Figs. 2 and 3), the maximum resonance amplitudes are observed. The first and second resonances, as well as the first self-oscillation zone, occur at the same frequencies as in the two previous cases, the amplitudes are, respectively, $Z_{r}=0.198, Z_{f}=0.033$ and $Z_{r}=1.9, Z_{f}=0.38$; the maximum amplitudes of the first self-oscillation zone are $Z_{r}=1.9, Z_{f}=0.38$. In this case, the third resonance peak also appears at a greater angular velocity occurring at $s=2.12$, with amplitudes $Z_{r}=2.93, Z_{f}=2.99$. Then, as before, at $s>3.41$, a self-oscillation zone is formed, where the amplitudes take on constant values $Z_{r}=0.15$ and $Z_{f}=0.0008$. The maximum amplitudes when one third of the cavity is fluid-filled and are associated with a specific behaviour of a "coupled" solid-fluid system [8], [19], and [23].

Furthermore, for cases $r_{0}=0.5 R, \quad r_{0}=0.333 R$ and $r_{0}=0.125 R$, frequencies of the first and second resonances, i.e., $s=0.065$ and $s=0.65$, as well as the frequencies of formation of self-oscillation zones, i.e., $0.065<s<0.65,0.65<s<3.41$ and $s>3.41$, do not change. The values of the first and second resonance amplitudes are damped. The third resonant amplitude is also damped, while the related resonance frequency is shifted to the right (see, e.g., Figs. 2 and 3), due to the increase of the fluid volume. At the same time, the fluid volume does have a significant effect on the amplitudes over the first, second and third zones of self-oscillations.

For an empty rotor, a breakdown of the amplitude-frequency characteristics of both the rotor and foundation, corresponding to the fundamental resonance, is typical for the studied cubic nonlinearity, as for a Duffing oscillator. This phenomenon, which arises as a result of a monotonic increase in the amplitudes and their sharp decrease after a certain frequency, has been studied in detail by many authors, e.g. [24] to [26]. In this case, the breakdown of the amplitude of the empty rotor with a gradual increase in frequency occurs at $Z_{r}=1.05$ and $s=1.33$, then the amplitudes of the rotor decrease to 0.15. A similar behaviour of the amplitude at $s=1.33$ is observed for the foundation; in this case, the maximum amplitude is $Z_{f}=0.115$, which is almost an order of magnitude less than the rotor amplitudes. The foundation amplitudes after the breakdown decrease to 0.01 . This difference between the amplitudes of the forced vibrations of the rotor and the foundation is primarily due to the ratio of the masses of the rotor and the foundation, as well as the value of linear eccentricity. As might be expected, an additional (first) linear resonance appears at $s=0.065$, according to the general theory in the book [27]. It should be noted that the third resonance is a specific feature of a non-linear system; this feature is addressed in greater detail below. For a fully fluid-filled rotor, the first and third resonances are suppressed, while the second resonance looks similar in a sense to the linearized case.

Thus, maximum amplitudes of forced oscillations of the rotor and the foundation are observed when one third of the rotor cavity is filled with fluid. In addition, a decrease in the fluid volume leads to a shift of the third resonance towards lower angular frequencies.

Decrease of the damping coefficient $(K=0.01)$ causes a shift of the third resonance to higher angular velocities (see Figs. 4 and 5). The first and second resonances are observed at virtually the same frequencies as for $r_{0}=0.667 R$ and $K=1$, i.e., at $s=0.065$ and $s=0.65$ with amplitudes $Z_{r}=0.62$, $Z_{f}=0.33$ and $Z_{r}=0.52, Z_{f}=0.07$. The first resonance at $K=0.01$ coincides in magnitude with the resonance at $K=1$. The second resonance of the rotor and foundation for $K=0.01$ is 6.35 and 54.28 times lower than the corresponding resonances for $K=1$. The number of self-oscillating zones does not change, i.e., three self-oscillation zones are observed, the first in the interval $0.065<s<0.65$, the second in the interval $0.65<s<3.41$, and the third at $s>3.41$ with maximum amplitudes $Z_{r}=0.43, Z_{f}=0.043, Z_{r}=0.9, Z_{f}=0.19$ and $Z_{r}=0.104, Z_{f}=0.017$, respectively. The shifted third resonance appears at $s=3.4$ with amplitudes $Z_{r}=8$ and $Z_{f}=13.3$, which is 2.76 times and 1.34 times less than the amplitudes of the third resonance of the rotor and foundation, respectively, at $K=1$.

With a significant increase in the damping coefficient, i.e., for $K=0.1$, the amplitudes of selfoscillations are damped and coincide with those at $K=1$. The resonance amplitudes of the rotor and the foundation increase in magnitude. The first, second and third resonances appear at the same frequencies as for $K=1$, that is, at $s=0.065, s=0.65$ and $s=2.12$, with amplitudes $Z_{r}=0.9, Z_{f}=0.3, Z=2.9, Z_{f}=4.1$ and $Z_{r}=25.5, Z_{f}=9.1$, which coincides in magnitude with 


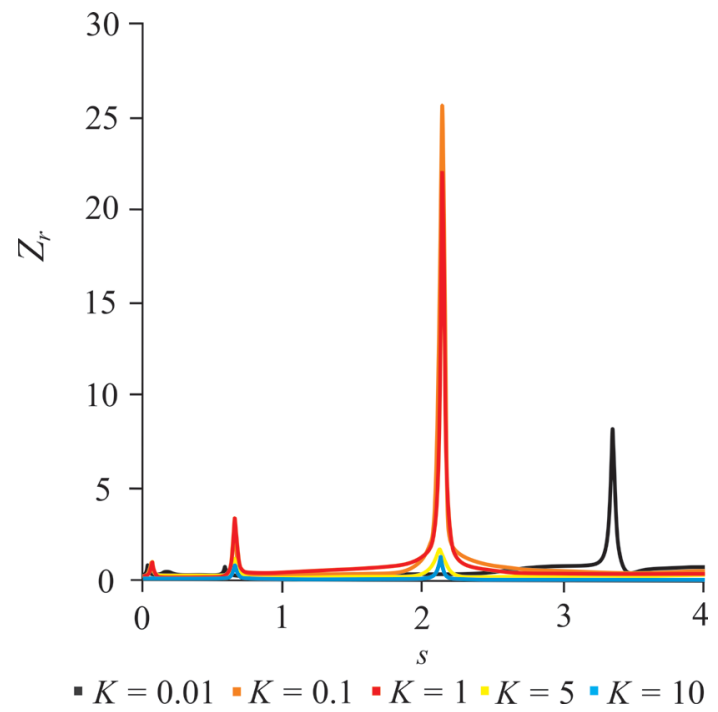

Fig. 4. Amplitude-frequency characteristics of the rotor $Z_{r}$ for various damping coefficients $K$

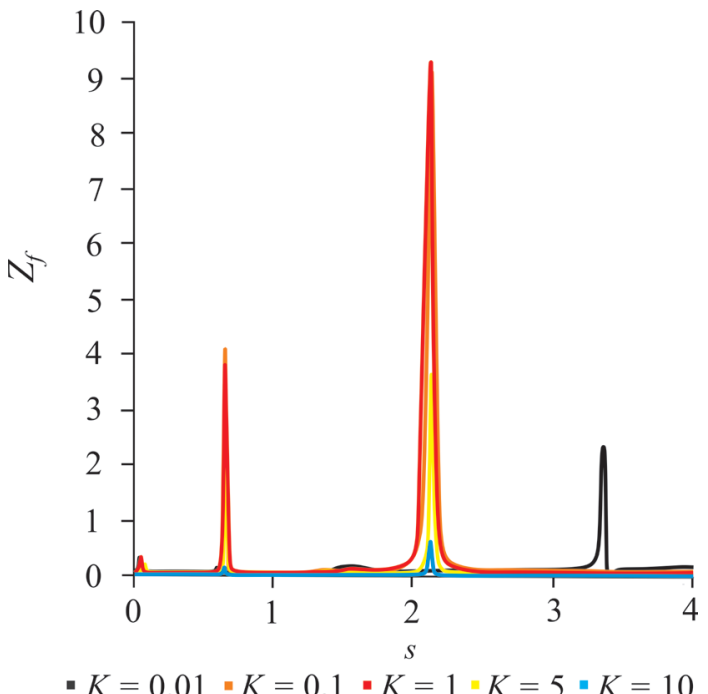

Fig. 5. Amplitude-frequency characteristics of the foundation $Z_{f}$ for various damping coefficients $K$

the first, second, and third amplitudes of both the rotor and the foundation for $K=1$.

Further increase in the damping coefficient (cases $K=5$ and $K=10$ ) leads to significant damping of selfoscillations and resonance amplitudes, for example, for $K=5$, the amplitudes of the first, second, and third resonances are equal to $Z_{r}=0.02, Z_{f}=0.003, Z_{r}=1.15$, $Z_{f}=0.4$ and $Z_{r}=1.7, Z_{f}=3.6$, respectively. For $K=10$, the amplitudes of the first, second, and third resonances are $Z_{r}=0.02, Z_{f}=0.003, Z_{r}=0.66, Z_{f}=0.1$ and $Z_{r}=1.1, Z_{f}=2.5$, respectively.

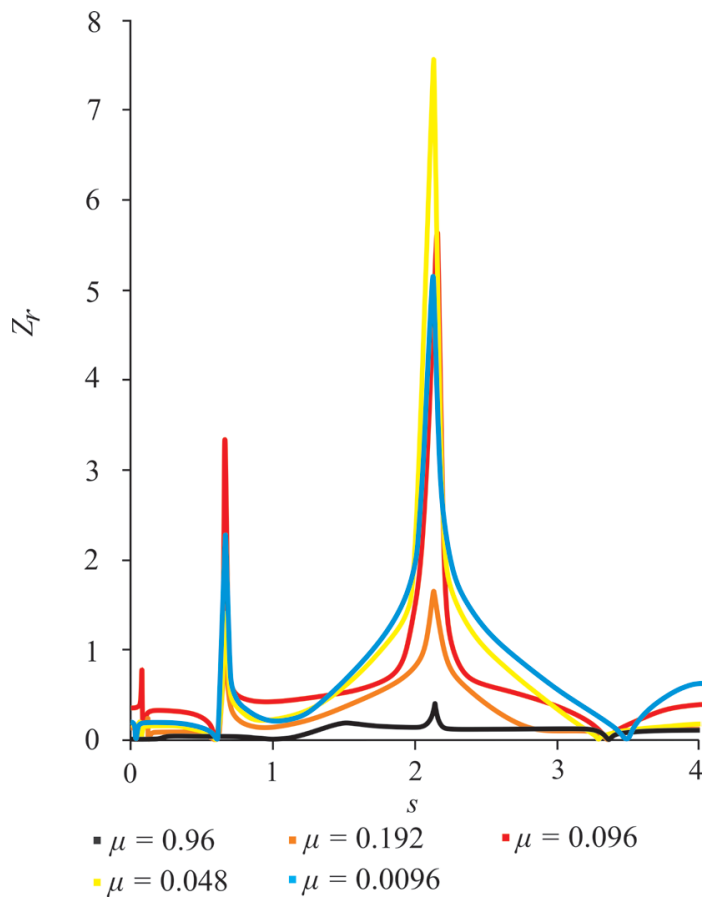

Fig. 6. Amplitude-frequency characteristics of the rotor $Z_{r}$ for different values of the foundation mass, $\mu$

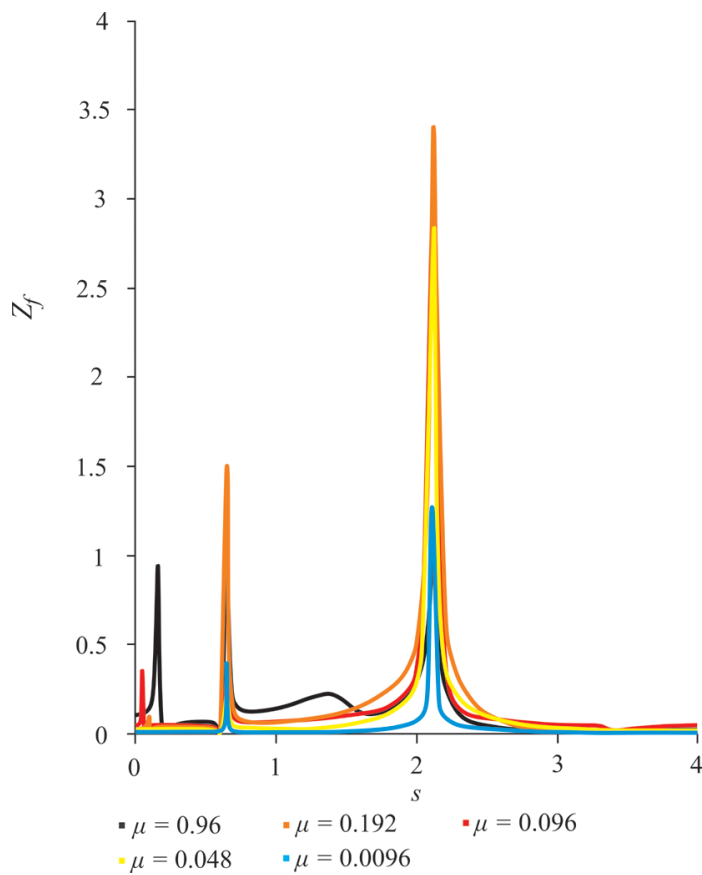

Fig. 7. Amplitude-frequency characteristics of the foundation $Z_{f}$ for different values of the foundation mass, $\mu$

In addition, with a significant decrease of the damping coefficient, a shift of the third resonance to higher angular velocities occurs. In particular, for a third fluid filled rotor, the aforementioned resonance 
is observed at the same frequency as that of an empty rotor $(s=3.4)$, which is a distinctive feature of nonlinear vibrations.

To assess the effect of the foundation mass on self-oscillations and resonance amplitudes at $r_{0}=0.667 R$, various ratios of the rotor and foundation masses $\mu=0.96, \mu=0.192, \mu=0.096, \mu=0.048$ and $\mu=0.0096$ were considered and for each case the amplitude-frequency characteristics were constructed (see Figs. 6 and 7).

In all of the above cases, the first, second, and third resonances were observed at $s=0.065, s=0.65$, and $s=2.12$, respectively. With an increase in the mass of the foundation (cases $\mu=0.096, \mu=0.048$ ), there is an increase in the amplitudes of the rotor at the third resonance (at $s=2.12, Z_{r}=5.6, Z_{f}=7.53$ ) and selfexcited vibrations in general, whereas the resonance amplitudes and amplitudes of self-oscillations of the foundation decrease in magnitude. In the case in which the mass of the foundation is slightly greater than the mass of the rotor (cases $\mu=0.192$ and $\mu=0.96$ ), the minimum amplitudes of the third resonances of both the rotor and the foundation are observed $\left(Z_{r}=1.94\right.$ and $Z_{f}=3.39, Z_{r}=0.34$ and $Z_{f}=1.01$ ). It should be noted that at $\mu=0.96$ in the interval $0.65<s<2.125$, a zone of intense self-oscillations of the foundation with a maximum amplitude of 0.28 appears (see Fig. 7). Damping the rotor amplitudes with a decrease in the mass of the foundation is a specific feature of the non-linear system. In the linear case, a decrease in the rotor amplitudes is observed with an increase in the mass of the foundation, as in this case the foundation itself acts as an anti-load.

To study the influence of nonlinear effects arising from nonlinearity of the restoring force, different values of the stiffness coefficient at the cubic term were considered, i.e., $C=0.01, C=0.1, C=1$, $C=10$ and $C=100$, and for each case the amplitudefrequency characteristics with a rotor fluid filled by one third, i.e., for $r_{0}=0.667 R$, are obtained (see Figs. 8 and 9).

An increase in the stiffness coefficient at the cubic term leads to a decrease in both the resonance amplitudes and the amplitudes of self-oscillations of the rotor and the foundation. The maximum amplitudes are observed in the case of $C=0.01$, for which the amplitudes of the first resonance for $s=0.065$ are $Z_{r}=1.86$ and $Z_{f}=0.15$, the amplitudes of the second resonance for $s=0.65$ are $Z_{r}=19.215$ and $Z_{f}=3.84$, and the amplitudes of the third resonance for $s=2.125$ are $Z_{r}=23.47$ and $Z_{f}=14.09$. The minimum resonance amplitudes are, respectively, observed in the case of $C=100$, where the amplitudes of the first

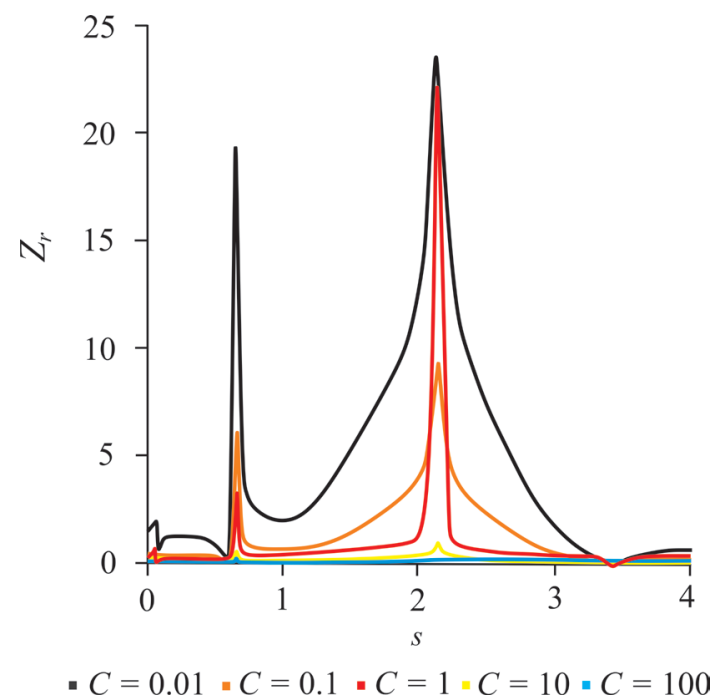

Fig. 8. Amplitude-frequency characteristics of the rotor $Z_{r}$ for different values of the stiffness coefficient of the cubic term, $C$

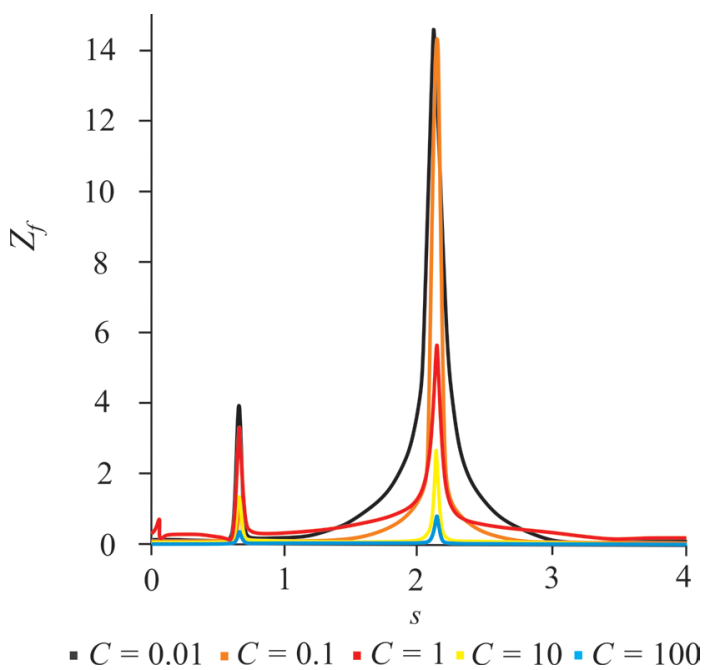

Fig. 9. Amplitude-frequency characteristics of the foundation $Z_{f}$ for different values of the stiffness coefficient of the cubic term, $C$

resonance for $s=0.065$ are equal to $Z_{r}=0.03$ and $Z_{f}=0.01$, the amplitudes of the second resonance for $s=0.65$ are equal to $Z_{r}=0.07$ and $Z_{f}=0.37$, and the amplitudes of the third resonance for $s=2.125$ are $Z_{r}=0.31$ and $Z_{f}=0.83$, i.e., with an increase in the stiffness coefficient by two orders of magnitude, the resonance amplitudes of the rotor and foundation decrease in 54.78 and 15 times for $s=0.065$, in 266.87 and 10.37 times for $s=0.65$, and in 76.21 and 16.97 times for $s=2.125$. Any significant shift of resonant frequencies and self-oscillation zones is observed when the stiffness coefficient changes. In this case, as in linear cases, an increase in stiffness leads to damping of all vibrations. Analysis of greater values 
of the stiffness coefficient is not of practical interest for the original engineering system.

Due to the imperfection of equipment and other factors at high operating speeds, it is important to determine the range of critical values of inbalance $e$, and to identify its impact on the behaviour of the system. In this work, the cases $E=0.5, E=1, E=5$ and $E=10$ were considered; for each case the amplitudefrequency characteristics were constructed (see Figs. 10 and 11).

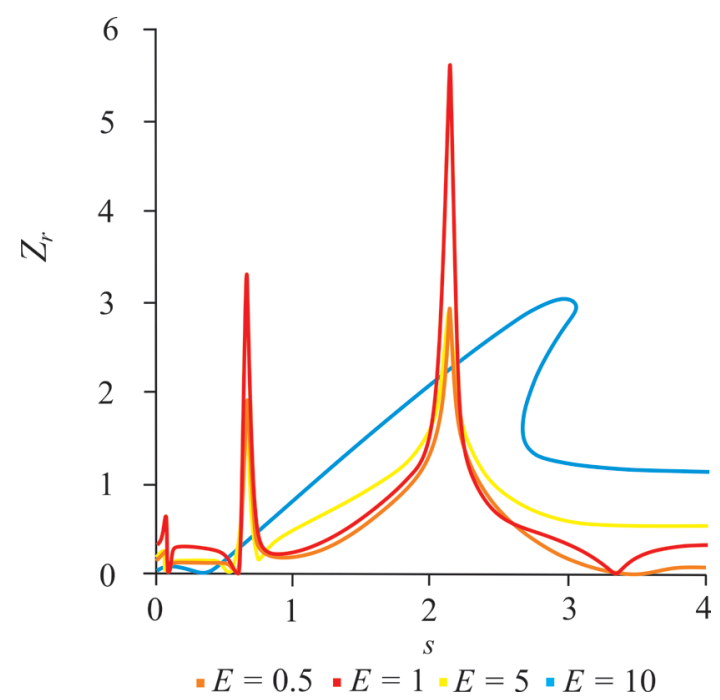

Fig. 10. Amplitude-frequency characteristics of the rotor $Z_{r}$ for different values of imbalance, $E$

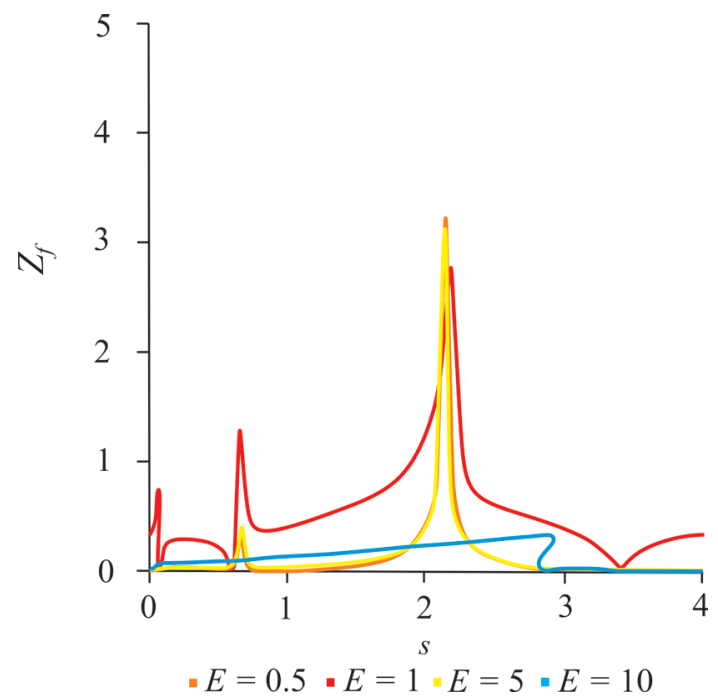

Fig. 11. Amplitude-frequency characteristics of the foundation $Z_{f}$ for different values of imbalance, $E$

For $E=0.5, E=1$ and $E=5$, as before, three resonance amplitudes are observed for $s=0.065$, $s=0.65$, and $s=2.125$. In this case, the change in the imbalance value practically does not affect the values of the resonance amplitudes and amplitudes of selfoscillations.

As the value of imbalance increases by an order of magnitude, i.e., at $E=10$, the amplitude-frequency characteristic of the rotor and the foundation is given by the nonlinearity of a hardening (stiff) type. Thus, with an increase in linear imbalance, self-oscillations arising from the action of the fluid are suppressed by forced vibrations. In this case, the characteristic breakdown of the amplitudes is shifted towards higher frequencies of the system. Further, after the breakdown, due to the self-centring effect, operation of the system is stabilized, and vibrations occur with an amplitude of about $Z_{r}=1.26$, which slightly exceeds the amplitude of self-oscillations. During rotor vibrations, one self-oscillation zone is observed in the interval $0<s<0.346$ with maximum amplitudes $Z_{r}=0.08$. During vibrations of the foundation, a sharp variation of the amplitude up to the value $Z_{f}=0.09$ is observed over the interval $0<s<0.112$. Further, with an increase in frequency, a gradual increase in foundation vibrations is observed until its amplitude breaks down, and, then, as in the case of the rotor, vibrations stabilize with an amplitude of about $Z_{f}=0.03$. In both cases, for the rotor and for the foundation, breakdown is observed at $s=2.817$.

To understand the nature of the first and third resonances, we construct the amplitude-frequency characteristics of the linear and non-linear "rotorfoundation" system without fluid (see Figs. 12 and 13), which can be obtained from the equations of motion, Eq. (13) for $F_{r}=0$ as

$$
\begin{aligned}
& \ddot{z}+n_{0}^{2}\left(z-z_{2}\right)+2 k \dot{z}=e \Omega_{0}^{2} \exp \left(i \Omega_{0} t\right), \\
& \ddot{z}_{2}+n_{2}^{2} z_{2}-n_{01}^{2}\left(z-z_{2}\right)+2 k_{0} \dot{z}_{2}=0,
\end{aligned}
$$

and

$$
\begin{aligned}
& \ddot{z}+n_{0}^{2}\left(z-z_{2}\right)+n_{1}\left(z-z_{2}\right)^{3}+2 k \dot{z}=e \Omega_{0}^{2} \exp \left(i \Omega_{0} t\right), \\
& \ddot{z}_{2}+n_{2}^{2} z_{2}-n_{01}^{2}\left(z-z_{2}\right)-n_{10}\left(z-z_{2}\right)^{3}+2 k_{0} \dot{z}_{2}=0 .
\end{aligned}
$$

The linear system of equations, Eq. (27) can be solved with sufficient degree of accuracy by numerical methods (see Figs. 12 and 13, black (solid) curve).

A cubic nonlinearity supports ultra-harmonic $\left(\Omega_{0}=3 \omega\right.$, or $\left.s=3\right)$-harmonic resonances $\left(\Omega_{0}=\omega / 2\right.$ and $\Omega_{0}=\omega / 3$, or $s=0.5$ and $\left.s=0.333\right)$. The frequencies of the latter are multiples of the fundamental one $\left(\Omega_{0}=\omega\right.$, or $\left.s=1\right)$. The solution of non-linear equations in Eq. (28) is found analytically, in order to incorporate explicitly the contribution of higher harmonics corresponding to sub- and ultra-harmonic oscillations. 


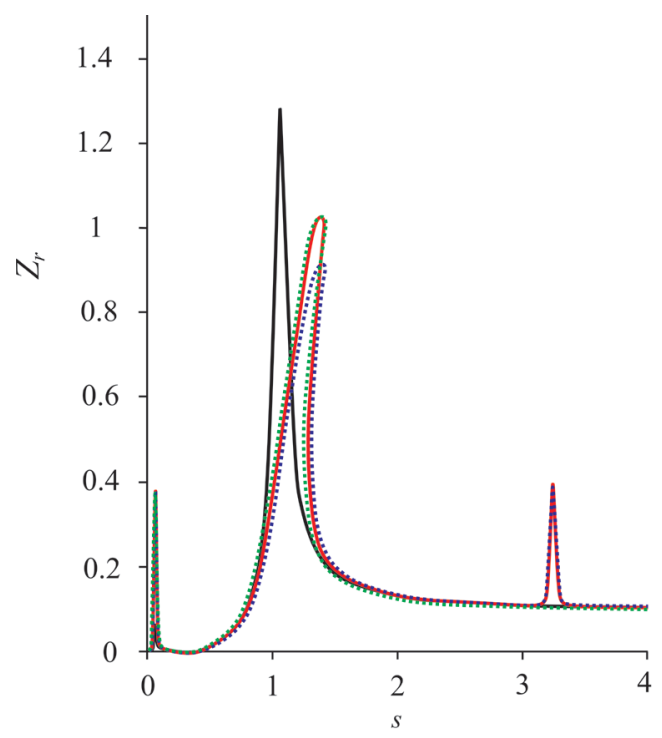

- Linear case numerical solution, - Nonlinear case analytical solution, - Nonlinear case numerical solution, " Nonlinear solution (subharmonic case)

Fig. 12. Amplitude-frequency characteristics of the rotor $Z_{r}$ in the linear and non-linear cases

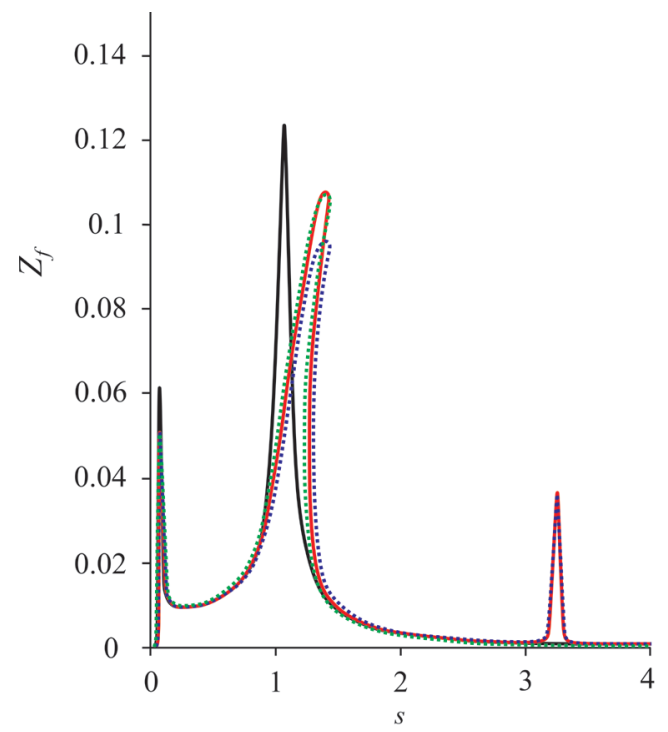

- Linear case numerical solution, - Nonlinear case analytical solution, " Nonlinear case numerical solution, " Nonlinear solution (subharmonic case)

Fig. 13. Amplitude-frequency characteristics of the foundation $Z_{f}$ in the linear and non-linear cases

The restoring force $F_{\mathrm{c}}$ approximated by Eq. (2) does not support sub-harmonic resonance in the system. Then, to find third-order sub-harmonic resonances, we introduce a notation $\Omega_{0}=3 \omega_{f},\left(\omega_{f}\right.$ is sub-harmonic frequency) and-search for a solution in the form [28]:

$$
z=\tilde{B}_{1 / 3} \exp \left(i\left(\omega_{f} t-\phi\right)\right)+\tilde{B}_{1} \exp \left(3 i\left(\omega_{f} t-\phi\right)\right),
$$

and

$$
z=\tilde{D}_{1 / 3} \exp \left(i\left(\omega_{f} t-\phi\right)\right)+\tilde{D}_{1} \exp \left(3 i\left(\omega_{f} t-\phi\right)\right) .
$$

Substituting Eqs. (29) and (30) into Eq. (28) and using the harmonic balance method, we obtain a system of algebraic equations:

$$
\begin{gathered}
\left(n_{0}^{2}-9 \omega_{f}^{2}\right) \tilde{B}_{1 / 3}-n_{0}^{2} \tilde{D}_{1 / 3}+\frac{3}{4} n_{1}\left(\tilde{B}_{1 / 3}-\tilde{D}_{1 / 3}\right)^{3}- \\
-\frac{3}{4} n_{1}\left(\tilde{B}_{1 / 3}-\tilde{D}_{1 / 3}\right)^{2}\left(\tilde{B}_{1}-\tilde{D}_{1}\right)- \\
-\frac{3}{2} n_{1}\left(\tilde{B}_{1 / 3}-\tilde{D}_{1 / 3}\right)\left(\tilde{B}_{1}-\tilde{D}_{1}\right)^{2}=9 e \omega_{f}^{2}, \\
\left(n_{0}^{2}-81 \omega_{f}^{2}\right) \tilde{B}_{1}-n_{0}^{2} \tilde{D}_{1}-\frac{1}{4} n_{1}\left(\tilde{B}_{1 / 3}-\tilde{D}_{1 / 3}\right)+ \\
+\frac{3}{2} n_{1}\left(\tilde{B}_{1 / 3}-\tilde{D}_{1 / 3}\right)^{2}\left(\tilde{B}_{1}-\tilde{D}_{1}\right)+\frac{3}{4} n_{1}\left(\tilde{B}_{1}-\tilde{D}_{1}\right)^{3}=0,( \\
\left(n_{2}^{2}+n_{01}^{2}-9 \omega_{f}^{2}\right) \tilde{D}_{1 / 3}-n_{01}^{2} \tilde{B}_{1 / 3}-\frac{3}{4} n_{10}\left(\tilde{B}_{1 / 3}-\tilde{D}_{1 / 3}\right)^{3}+ \\
+\frac{3}{4} n_{10}\left(\tilde{B}_{1 / 3}-\tilde{D}_{1 / 3}\right)^{2}\left(\tilde{B}_{1}-\tilde{D}_{1}\right)+ \\
+\frac{3}{2} n_{10}\left(\tilde{B}_{1 / 3}-\tilde{D}_{1 / 3}\right)\left(\tilde{B}_{1}-\tilde{D}_{1}\right)^{2}=0, \\
\left(n_{2}^{2}+n_{01}^{2}-81 \omega_{f}^{2}\right) \tilde{D}_{1}-n_{01}^{2} \tilde{B}_{1}+\frac{1}{4} n_{10}\left(\tilde{B}_{1 / 3}-\tilde{D}_{1 / 3}\right)- \\
-\frac{3}{2} n_{10}\left(\tilde{B}_{1 / 3}-\tilde{D}_{1 / 3}\right)^{2}\left(\tilde{B}_{1}-\tilde{D}_{1}\right)-\frac{3}{4} n_{10}\left(\tilde{B}_{1}-\tilde{D}_{1}\right)^{3}=0 .
\end{gathered}
$$

Solving Eqs. (31) to (34), we obtain the amplitudes of the steady-state forced vibrations $\tilde{B}_{1 / 3}, \tilde{B}_{1}, \tilde{D}_{1 / 3}$ and $\tilde{D}_{1}$, which can be used to construct the amplitude-frequency characteristics of the rotor and foundation in the case of sub-harmonic vibrations.

To determine ultra-harmonic resonances, the solution is sought in the form:

$$
z=Z_{r} \exp \left(i\left(\Omega_{0} t-\phi\right)\right)+B \exp \left(3 i\left(\Omega_{0} t-\phi\right)\right),
$$

and

$$
z_{2}=Z_{f} \exp \left(i\left(\Omega_{0} t-\phi\right)\right)+D \exp \left(3 i\left(\Omega_{0} t-\phi\right)\right) .
$$

In this case, the force of reaction of the fluid on the rotor wall has the form:

$$
\begin{aligned}
F_{r}= & m_{L} \Omega_{0}{ }^{2} A \exp \left(i\left(\Omega_{0} t-\phi\right)\right)+ \\
& +9 m_{L} \Omega_{0}{ }^{2} B \exp \left(3 i\left(\Omega_{0} t-\phi\right)\right) .
\end{aligned}
$$

Substituting Eqs. (35) and (36) into Eq. (4) and using the harmonic balance method, we obtain a system of non-linear algebraic equations resulting in 
and

$$
B=d_{0} D,
$$

where

$$
D=d B
$$

$$
\begin{gathered}
d_{0}=a_{0}+i a_{1}, \quad d d_{0}=1, \quad d=\frac{a_{0}-i a_{1}}{a_{0}^{2}+a_{1}^{2}}, \\
a_{0}=\frac{-\left(b_{0} c_{0}+b_{1} c_{1}\right)}{b_{3}}, \quad a_{1}=\frac{-\left(b_{0} c_{1}-b_{1} c_{0}\right)}{b_{3}}, \\
b_{3}=b_{0}^{2}+b_{1}^{2}, \quad b_{0}=-9 \Omega_{0}^{2} \mu\left(1+\mu_{L} D_{0}\right), \\
b_{1}=6 \Omega_{0} k \mu, \quad D_{0}=\frac{1}{4 \gamma-3}, \quad c_{0}=n_{2}^{2}-9 \Omega_{0}^{2}, \\
c_{1}=6 \Omega_{0} k_{0}, \quad b_{1}=6 \Omega_{0} k \mu .
\end{gathered}
$$

To determine the unknowns, we also have a system of two equations, which takes the form:

$$
\begin{aligned}
e_{0} C- & n_{01}\left(Z_{r}-Z_{f}\right)+n_{10}\left(Z_{r}-Z_{f}\right) \cdot\left(\frac{3}{4}\left(Z_{r}-Z_{f}\right)^{2}+\right. \\
+ & \left.\frac{3}{2}(B-D)^{2}+\frac{3}{4}(B-D)\left(Z_{r}-Z_{f}\right)\right)=0, \\
& e_{1} D-n_{01}(B-D)-n_{10} \cdot\left(\frac{3}{4}(B-D)^{3}+\right. \\
+ & \left.\frac{3}{2}\left(Z_{r}-Z_{f}\right)^{2}(B-D)+\frac{1}{4}\left(Z_{r}-Z_{f}\right)^{3}\right)=0,
\end{aligned}
$$

where

$$
e_{0}=n_{2}^{2}-\Omega_{0}{ }^{2}+i 2 k_{0} \Omega_{0}, \quad e_{1}=n_{2}^{2}-9 \Omega_{0}{ }^{2}+i 6 k_{0} \Omega_{0} .
$$

From the system in Eq. (40), taking into account Eqs. (38) and (39), we have:

where

$$
B^{2}+g B+l=0
$$

$$
g=\frac{f_{2}}{2(1-d)}, \quad l=\frac{-e_{01} f+f_{1}\left(f_{2} n_{01}+\frac{3}{4} n_{10} f_{2}^{3}\right)}{\frac{3}{2} f_{2} f_{1} n_{01}(1-d)^{2}} .
$$

Solving Eq. (41), we obtain the values of the amplitudes $B_{1,2}$, and using Eqs. (38) to (40), we can find all the amplitudes of ultra-harmonic oscillations and the phase angle.

Thus, by comparing the amplitude-frequency characteristics obtained from the system in Eq. (27) and the amplitude-frequency characteristics obtained from systems in Eqs. (4) and (28), it is possible to determine whether sub-harmonic and ultra-harmonic resonances are present.

As sub-harmonic and ultra-harmonic resonances are observed only in non-linear systems, and (as in the linear case) there is no third resonance, which is observed at $s=3.24$ (see Figs. 12 and 13, black (solid) curve), we can conclude that this resonance is ultra-harmonic, which is also confirmed by the fact that $\Omega_{0} \approx 3 \omega$; in doing so, a shift in frequency is due foundation vibrations, whereas the presence of the first resonance is typical for a multi-degree of freedom system.

The numerical solution of the non-linear system in Eq. (28) does not indicate the presence of subharmonic resonances (see Figs. 12 and 13, purple curve).

To design rotor installation with these parameters, the optimal value of the foundation mass has to be up to 1.042 of the rotor mass, with linear eccentricity not exceeding $E=10$. It is recommended to increase the stiffness coefficient at the cubic term by five orders of magnitude relative to the linear term and to decrease the damping coefficient by two orders, which will result in smaller amplitudes and will increase ultraharmonic resonances.

Thus, for stable operation of the system at any filling of the cavity, it is necessary that the operating speed is in the range $0.065<s<0.65$ or $s>3.41$.

\section{CONCLUSIONS}

A method for calculating amplitudes and constructing frequency characteristics of forced and self-excited vibrations of the "rotor-fluid-foundation" system on rolling bearings with a non-linear characteristic based on the method of complex amplitudes and harmonic balance has been developed. The specific features of the non-linear dynamic behaviour characterised by ultra-harmonic vibrations are studied. The optimal parameters of linear imbalance, the mass of the foundation, the fluid volume in the rotor cavity, the stiffness and damping coefficients, for which the amplitudes take optimal values, have been determined.

\section{ACKNOWLEDGEMENTS}

This work has been supported financially by the research project AP08856167 of the Ministry of Education and Science of the Republic of Kazakhstan and was performed at Research Institute of Mathematics and Mechanics in Al-Farabi Kazakh National University, which is gratefully acknowledged by the authors. The corresponding author gratefully acknowledges financial support from the Slovenian Research Agency (ARRS), Grant Ref. J2-9224. 


\section{REFERENCES}

[1] Sharma, A., Upadhyay, N., Kankar, P. K., Amarnath, M. (2018). Non-linear dynamic investigations on rolling element bearings: A review. Advances in Mechanical Engineering, vol. 10, no. 3, p. 1-15, DOI:10.1177/1687814018764148.

[2] Wang, G., Yuan, H., Sun, H. (2020). An investigation on the instability of a flexible liquid-filled rotor. Proceedings of the Institution of Mechanical Engineers, Part G: Journal of Aerospace Engineering, vol. 234, no. 2, p. 165-172, DOl:10.1177/0954410019854262.

[3] Zhang, X., Han, Q., Peng, Z., Chu, F. (2013). Stability analysis of a rotor-bearing system with time-varying bearing stiffness due to finite number of balls and unbalanced force. Journal of Sound and Vibration, vol. 332, no. 25, p. 6768-6784, DOI:10.1016/j.jsv.2013.08.002.

[4] Kumar Avshist, R., Peng, Q. (2018). Non-linear dynamic modeling of the cracked rotor ball bearing system with emphasis on damage detection capabilities. Journal of Vibration and Acoustics, vol. 140, no. 4, p. 041018-1-04101810, Dol:10.1115/1.4039404.

[5] Li, Z., Li, J., Li, M. (2018). Non-linear dynamics of unsymmetrical rotor-bearing system with fault of parallel misalignment. Advances in Mechanical Engineering, vol. 10, no. 5, p. 1-17, Dol:10.1177/1687814018772908.

[6] Xu, Q., Niu, J., Yao, H., Zhao, L., Wen, B. (2019). Nonlinear dynamic behavior and stability of a rotor/seal system with the dynamic vibration absorber. Advances in Mechanical Engineering, vol. 11, no. 1, p. 1-17, DOI:10.1177/1687814018819578.

[7] Nan, G., Tang, M., Chen, E., Yang, A. (2016). Non-linear dynamic mechanism of rolling element bearings with an internal clearance in a rotor-bearing system. Advances in Mechanical Engineering, vol. 8, no. 11, p. 1-9, DOI:10.1177/1687814016679588.

[8] Kydyrbekuly, A., Khajiyeva, L., Ybraev, G.G.A.Y., Kaplunov, J. (2016). Non-linear vibrations of a rotor-fluid-foundation system supported by rolling bearings. Strojniški vestnik Journal of Mechanical Engineering, vol. 62, no. 6, p. 351-362, DOI:10.5545/sv-jme.2016.3423.

[9] Ishida, Y., Liu, J. (2010). Elimination of unstable ranges of rotors utilizing discontinuous spring characteristics: An asymmetrical shaft system, an asymmetrical rotor system, and a rotor system with liquid. Journal of Vibration and Acoustics, vol. 132, no. 1, p. 011011-1-011011-8, DOI:10.1115/1.4000842.

[10] Xia, Z., Qiao, G., Zheng, T., Zhang, W. (2009). Non-linear modelling and dynamic analysis of the rotor-bearing system. Nonlinear Dynamics, vol. 57, no. 4, p. 559-577, D0l:10.1007/ s11071-008-9442-3.

[11] Luczko, J. (2002). A geometrically non-linear model of rotating shafts with internal resonance and self-excited vibrations. Journal of Sound and Vibrations, vol. 255, no. 3, p. 433-456, DOI:10.1006/jsvi.2001.4164.
[12] Friswell, M., Penny, J., Garvey, S., \& Lees, A. (2010). Dynamics of Rotating Machines (Cambridge Aerospace Series). Cambridge University Press, Cambridge, D0l:10.1017/ CB09780511780509.

[13] Vance, J.M., Zeidan, F.Y., Murphy, B. (2010). Machinery Vibration and Rotordynamics. John Wiley \& Sons, Inc., Hoboken, DOI:10.1002/9780470903704.

[14] Harris, T.A. (2001). Rolling Bearing Analysis. John Wiley \& Sons, Inc., Hoboken

[15] David, P.F., Poplawski, J.V. (2004). Transient vibration prediction for rotors on ball bearings using loaddependent non-linear bearing stiffness. International Journal of Rotating Machinery, vol. 10, no. 6, p. 489-494, DOI:10.1080/10236210490504102.

[16] Yamamoto, T., Ishida, Y. (1977). Theoretical discussions on vibrations of a rotating shaft with non-linear spring characteristics. Ingenieur-Archiv, vol. 46, no. 2, p. 125-135, DOI:10.1007/BF00538746.

[17] Changsen, W. (1991). Analysis of Rolling Element Bearings. Mechanical Engineering Publications Limited.

[18] Jin, Y., Lu, Z., Yang, R., Hou, L., Chen, Y. (2018). A new nonlinear force model to replace the Hertzian contact model in a rigid-rotor ball bearing system. Applied Mathematics and Mechanics, vol. 39, no. 3, p. 365-378, D0l:10.1007/s10483 018-2308-9.

[19] Ishida, Y., Yamamoto, T. (2013). Linear and Nonlinear Rotordynamics: A Modern Treatment with Applications. John Wiley \& Sons, Weinheim, D0l:10.1002/9783527651894.

[20] Isayuk-Sayevskaya, A.R., Kelzon, A.S. (1995). Dynamics of a high-revolution scroll centrifuge. Machine Vibration, vol. 3, no. 4, p. 233-237.

[21] Tondl, A. (1965). Some Problems of Rotor Dynamics. Chapman and Hall, London.

[22] Rao, S. (2018). Mechanical Vibrations. Pearson Education, Inc., London.

[23] Kollmann, F.G. (1962). Experimentelle und theoretische Untersuchungen uber die kritischen Drehzahlen flussigkeitsgefullter Hohlkorper. Forschung auf dem Gebiete des Ingenieurwesens A, vol. 28, no. 4,p. 115-123, vol. 28, no. 5, p. 147-153, D0l:10.1007/BF02557449.

[24] Schmidt, G., Tondl, A. (1986). Non-linear Vibrations. Cambridge University Press, Cambridge, D0l:10.1017/ СB09780511735752.

[25] Kovacic, I., Brennan, J. (2011). The Duffing Equation: Nonlinear Oscillators and Their Behaviour. John Wiley \& Sons, Hoboken, DOI:10.1002/9780470977859.

[26] Cveticanin, L. (2018). Strong Non-linear Oscillators. Springer, Cham, Dol:10.1007/978-3-319-58826-1.

[27] Krämer, E. (1993). Dynamics of Rotors and Foundations, Springer-Verlag, New York, Dol:10.1007/978-3-662-02798-1.

[28] Hayashi, C. (2014). Non-linear Oscillations in Physical Systems. Princeton University Press, Princeton. 